\title{
FACTORES IMPLICADOS EN LA ADOPCIÓN DE SOFTWARE LIBRE EN LAS PYME DE MEDELLÍN
}

\author{
Factors involved in the adoption of free software by SMEs in Medellín \\ Jorge Andrés Moreno-Agudelo \\ Administrador Tecnológico, Instituto Tecnológico Metropolitano ITM, Medellín-Colombia, \\ morenojorge28@gmail.com
}

Jhoany Alejandro Valencia-Arias

MSc en Ingeniería de Sistemas, Instituto Tecnológico Metropolitano ITM, Medellín-Colombia, jhoanyvalencia@itm.edu.co

\begin{abstract}
Cómo citar / How to cite
Moreno-Agudelo, J. A. y Valencia-Arias, J. A. (2017). Factores implicados en la adopción de software libre en las Pyme de Medellín. Revista CEA, 3(6), 55-75.
\end{abstract}

Recibido: 10 de febrero de 2017

Aceptado: 15 de mayo de 2017

\section{Resumen}

El software de código abierto trae beneficios para las Pyme en economías emergentes, como ahorro en costos de adquisición, modificación del código fuente, independencia del proveedor, mayor calidad y seguridad del software. El objetivo de este artículo es identificar los factores de los adoptantes y no adoptantes de software libre y estructurar estrategias que fomenten la adopción en las Pyme de la ciudad de Medellín, a través de una entrevista a gerentes y jefes de las áreas de sistemas.

Una de las conclusiones es que la cultura tecnológica de la organización y su infraestructura son factores que determinan la facilidad de su uso. Que la calidad del software es un determinante para percibir la utilidad del software libre y que tanto el apoyo gubernamental como la disponibilidad de personal capacitado en TIC son factores que influyen en la actitud que toma la empresa frente al uso de estas tecnologías libres.
Palabras clave: software libre, software libre en Pyme, modelo de aceptación tecnológica, adopción de software libre, software de código abierto.

\begin{abstract}
In emerging economies, open source software brings SMEs benefits such as purchase cost savings, source code modification, vendor independence, higher quality and more secure software. The objective of this article is to identify the factors that drive adopters and non-adopters of free software and develop strategies that encourage its adoption at SMEs in Medellín by means of interviews with CEOs and IT department directors. One of the conclusions is that the technological culture of the organization and its infrastructure are factors that determine the ease of use. Besides, software quality is a decisive element in the perception of the usefulness of free software. Finally, both government support and the availability of ICT-trained personnel are factors that influence the attitude of a company towards the usage of these free technologies.
\end{abstract}


Keywords: free software, free software in SMEs, technology acceptance model, free software adoption, open source software.

\section{INTRODUCCIÓN}

El Software de Código Abierto (OSS) se ha extendido en los últimos años (Urban \& Greyling, 2015) ganando atención a nivel mundial (Bandi, Kanamadi \& Angadi, 2016) y convirtiéndose en una de las principales fuerzas motrices del ecosistema empresarial (Silic \& Back, 2016).

Es importante tener presente que el uso de software libre es gratuito (Roca, 2007), por lo cual resulta ventajoso su implementación en las Pyme, ya que se convierte en una manera de aminorar la brecha respecto a las grandes empresas, sin necesidad de hacer una gran inversión (Galvis, 2008). En los últimos años, el software de código abierto ha pasado a ser una corriente principal y ha madurado, al punto en que existen grandes cantidades de software para diferentes tipos de negocio, esto se evidencia en que el $46 \%$ de las empresas norteamericanas utilicen software de código abierto (Hammond, 2010 citado Lakka, 2011).

El acelerado ritmo de los nuevos avances en las Tecnologías de la Información y de la Comunicación (TIC) representa un desafió continuo para los países en desarrollo, en la adquisición de tecnologías que le permitan funcionar y competir en el mercado globalizado (Hoyos y Valencia, 2011). Esto representa un problema importante, porque los países en desarrollo a menudo carecen de los recursos financieros y humanos que son necesarios en las empresas para fomentar infraestructuras TIC eficientes. Dado lo anterior, el software libre presenta a los países en desarrollo la oportunidad de superar algunos de los problemas tecnológicos y reducir la brecha digital a un costo relativamente bajo (Ellis \& Van Belle, 2009).

En el contexto colombiano, el uso de las TIC presenta un bajo nivel, ya que el $87 \%$ de las empresas las utilizan en un nivel 1 (uso de operaciones básicas) representadas principalmente en las Pyme, lo que puede indicar que a mayores recursos como equipos de cómputo y mayor tamaño en la estructura organizacional se hace más evidente el uso de las TIC, en contraposición con las pequeñas empresas que, al tener menor recurso y personal, la adopción de TIC es menor (Ortega Ruiz, 2014). En las Pyme de Colombia aún es muy bajo el grado de disponibilidad y utilización las TIC, solo el $7 \%$ de estas empresas utiliza las TIC, debido a la baja penetración de equipos de cómputo en sus procesos, lo que evidencia una fuerte brecha digital y un bajo nivel de uso. Aunque puede haber ventajas de adoptar software libre, especialmente para las pequeñas empresas en los mercados emergentes, existen una serie de barreras. La falta de conocimiento y la publicidad de este tipo de sistemas son fundamentales para la lenta difusión de OSS. Los factores clave que contribuyen a la falta de conocimiento del producto son la falta de comercialización OSS y proveedores de OSS de fácil acceso (Ellis \& Van Belle, 2009).

En Colombia, según la Cámara Colombiana de la Informativa y las Telecomunicaciones, solo el $36 \%$ de las empresas Pyme del país hacen un uso adecuado de las tecnologías de la información (Yohai, 2013). Lo anterior lleva a las siguientes preguntas: ¿Qué factores han permitido que estas Pyme implementen el software libre en sus procesos organizacionales? ¿Qué condiciones han hecho que el restante $64 \%$ no lo implemente, a pesar de los beneficios en costo y operación? Este panorama contrasta con el contexto de las Pyme en los países desarrollados, donde las pequeñas empresas se han apropiado de 
recursos gratuitos para mejorar sus ingresos y reducir costos, por lo que se hace necesario abordar estas preguntas desde el contexto de la gestión tecnológica y aportar al conocimiento científico sobre los factores implicados en la adopción de software libre en el contexto de economías emergentes, lo cual puede servir como insumo para validar futuros modelos de adopción de este tipo de tecnologías.

Para esta investigación se toma la ciudad de Medellín como foco de la investigación, debido a que cuenta con el clúster «Tecnología, Información y Comunicación -TIC», el cual se articula con el plan de ciencia y tecnología e innovación de la ciudad, desde donde se desarrollan programas y contenidos en torno a la promoción de la gestión tecnológica, la internacionalización, acceso a mercados y capital, cultura y plataformas de innovación, tomando entre sus iniciativas la modernización de las plataformas organizacionales y administrativas de las empresas establecidas en la ciudad. Por lo tanto, se observa un ecosistema empresarial favorable para la adopción de nuevas tecnologías, como es el caso del software libre.

Para el desarrollo de esta investigación se realiza un marco teórico en el que se revisan documentos científicos sobre el uso y adopción de software libre, ventajas y desventajas para las empresas Pyme; se estudian los factores externos implicados en este proceso descritos por varios autores y el modelo de aceptación tecnológica en el cual se basa la investigación. Por último, se hace un análisis de los resultados obtenidos por medio de mapas mentales, que explican los factores que inciden en la adopción de software libre por parte de las Pyme en la ciudad de Medellín y se expone de manera detallada, con el fin de explorar qué elementos hacen que los empleados adopten el software libre en sus actividades laborales, y finalmente poder proponer estrategias de adopción de software libre en las pequeñas y medianas empresas de la ciudad de Medellín.

\section{MARCO TEÓRICO}

\section{Software libre en Pyme}

El OSS (Open Source Software o software de código abierto) puede ser definido como el software que permite al usuario modificar el código fuente, así como corregir los errores y personalizar la aplicación para los requisitos específicos que los requiera (Johnston \& Seymour, 2005 citado en Ellis \& Van Belle, 2009). La disponibilidad y la apertura del código de fuente de sistemas OSS permite a las comunidades de usuarios, desarrolladores, patrocinadores y académicos, desarrollar, rediseñar y validar los softwares libres de forma independiente, mejorando su funcionalidad, valor y calidad.

El software de código abierto ha sido considerado como un fenómeno creciente (Shaikh, 2016), lo que lo ha posicionado como un tema altamente debatido en la industria del software, tanto teórica como empíricamente (Morgan, \& Finnegan, 2007). La importancia del software de código abierto se ha visto reflejada gracias a su capacidad de generar un mayor valor a los usuarios y conducir a mayores ingresos para las empresas (Garmabaki, Ahmadi, Mahdavi \& Ahmadi, 2015), otorgándoles, adicionalmente, beneficios de apertura e innovación (Méndez, 2016). A lo largo de este marco, se mostrará la utilidad tanto para las empresas como para los individuos.

OSS ha atraído millones de usuarios y desarrolladores (Alexy, Henkel \& Wallin, 2013), en la actualidad existen populares softwares de código abierto como Apache, Linux, Mozilla Firefox, OpenOffice, Skype (Kamseu \& Habra, 2009; Whitmore, Choi \& Arzrumtsyan, 2015), 
WordPress, VLC, entre otros (Kagiri, He \& Henglin, 2013), que son reconocidos por los usuarios y por el mundo empresarial (Kamseu \& Habra, 2009).

Uribe (2007), hace una clasificación del software libre de acuerdo con unas características:

- Según el costo: puede ser de las dos clases, es decir, de costo cero o de costo mayor que cero, lo que lo diferencia del software propietario es que su costo es independiente del número de computadoras que se poseen.

- Según la apertura del código de fuente: software open source, es decir, de código abierto o por el contrario con su código de fuente cerrado sin derecho a modificación.

- Según su protección: el software libre siempre está protegido con licencias, y más específicamente, con licencias de copyleft (que permite modificar un programa, pero exigiendo que siga siendo libre).

Tanto las empresas como los individuos enfrentan diversas dificultades y desafíos para dar el paso hacia la adopción de OSS; las organizaciones no solo tienen que resolver retos derivados de los componentes tecnológicos, sino también de los cambios que tienen que enfrentar en lo concerniente a la cultura organizacional y transformación de la mentalidad de los responsables de la toma de decisiones de TI (Méndez, 2016).

Actualmente existen grandes cantidades de software libre para diferentes tipos de negocio. La adopción de este tipo de software se ha visto en aumento; el Apache, un servidor web ha crecido $7.26 \%$ en los últimos cuatro años hasta el 2011 (Macredie y Mijinyawa, 2011) y Linux creció en la ejecución en equipos entre un 20 \% y un $27 \%$ entre los años 2007 y 2009. Estos datos también son reflejados en encuestas realizadas en el sector público, atención de la salud, grandes empresas y economías en desarrollo; la rápida expansión en el uso de este tipo de software libre y en diferentes entornos hace que sea importante para los investigadores ahondar en temas de adopción de OSS (Macredie y Mijinyawa, 2011).

A pesar de su importancia, en el contexto de las economías emergentes se presenta un problema importante, porque los países en desarrollo a menudo carecen de los recursos financieros y humanos que son necesarios en las empresas para fomentar infraestructuras TIC eficientes. En Colombia aún es muy bajo el nivel de adquisición y uso de las TIC en las empresas Pyme; ello vislumbra una importante desventaja competitiva en un entorno que demanda estar preparados para participar en un mercado que está cada vez más interconectado, tanto a nivel nacional como internacional (Gálvez, Riascos \& Contreras, 2014), esto se hace más complejo al evidenciar que solo el $36 \%$ de las Pymes utiliza de manera adecuada las tecnologías de la información (Caracol Radio, 2013), teniendo en cuenta que ni el tamaño ni la edad de la empresa aparecen como factores que inciden de manera considerable en la influencia de las TIC en ambiente web sobre el rendimiento de la organización, por lo cual, teniendo en cuenta dichos factores, las Pyme tienen en general las mismas oportunidades de beneficiarse de estas herramientas y prácticas (Gálvez et al., 2014). Es pertinente concientizar a los empresarios de la situación señalada para que participen de programas gubernamentales de apoyo para la implementación y aprovechamiento de estas herramientas, tales como Mipyme Digital y de otros privados que existen en gremios $y$ universidades.

Observando este amplio uso de software libre por parte de grandes organizaciones colombianas implementado en procesos claves; para el caso de las Pyme en los mercados emergentes, se encuentran una 
serie de barreras en estudios como el de Ellis y Van Belle (2009), que plantea que existe un poco publicidad y falta de conocimiento de los sistemas libres.

Como factor clave en la falta de conocimiento de estos sistemas, se encuentran la poca disponibilidad de proveedores especializados en software libre y la baja comercialización que existe. En concordancia con este estudio realizado, se encuentra en la investigación de Uribe (2007), que el $50 \%$ de las empresas bajo este análisis consideran que el mayor obstáculo es la falta de formación técnica, un 20 \% la falta apoyo directivo, y lejos coincidieron con un $10 \%$ la falta de costo, poco entendimiento de software libre y falta de recursos. Por otro lado, las empresas que no utilizan software libre respondieron que no lo hacen por la falta de formación técnica y de apoyo directivo. Las que no lo utilizan es por falta de conocimiento, lo que hace fundamental el desarrollo de eventos de promoción del software libre.

Bajo estas causas coinciden autores como Ellis y Van Belle (2009), quienes establecen que hay una falta general de conocimiento en software libre y en software de código abierto, y que esto influye en la disponibilidad de personal técnico experto en estos sistemas, que además es visto como un factor importante para la disminución de la adopción del software libre entre las pequeñas empresas. Adicional a esto, la dificultad en el intercambio de archivos que se da frecuentemente en los documentos de texto, ya que si se quieren abrir con un software libre aparecerá error o se perderán datos y problemas de implantación e interoperabilidad, dado que el software constituye algo nuevo, ello supone afrontar un costo de aprendizaje de instalación, de migración y de interoperabilidad siendo necesario aclarar que el costo de migración está referido al software, ya que en lo que respecta a hardware, generalmente el software libre no posee mayores requerimientos que el software propietario (Uribe, 2007).

En este mismo contexto, Colombia como parte de los compromisos internacionales adquiridos por el Gobierno Nacional, en la Declaración del Milenio, viene adelantando esfuerzos por reducir la brecha digital existente, tanto entre los usuarios naturales como en los empresariales, y ha desarrollado como estrategia la elaboración de un plan de trabajo (Ortega, 2016). Para impulsar el uso de estas herramientas en las Pyme de Colombia, el Gobierno juega un papel importante con programas desde su plan estratégico, como el «Plan Vive Digital Colombia» del Ministerio de Tecnologías de la Información y Comunicación desde el 2010 y Mipyme Vive Digital del 2014; en los que se plantea el impulso al desarrollo de aplicaciones y uso de TIC, impulsar el fortalecimiento de cadenas productivas en las Pyme a través de las TIC, así como divulgar y socializar el uso de estas tecnologías en las Pyme; se hace importante encontrar una herramienta que ayude a aminorar la brecha tecnológica y de competitividad que existe entre las grandes empresas y las Pyme, sin hacer una gran inversión y que se alcance el objetivo de aumentar la competitividad y la productividad. El Gobierno se plantea eliminar barreras percibidas por los empresarios como, «las TIC son costosas», "traen más gastos», "son para empresas grandes», planteadas en el estudio de caracterización sobre la relación de las Pyme y las TIC en 2016.

Complementando estas barreras que las Pyme encuentran para la implementación de software libre, Bonet (2007) indica que los problemas identificados están relacionados con la piratería, el miedo al cambio y la desconfianza. En España, el índice de piratería se sitúa en el $46 \%$, lo que representa unas pérdidas para el sector TIC de unos 600 millones de euros. Algunas empresas tienden a confundir el software libre con el gratuito y 
algunas compañías descartan su implantación, bien porque no hay software libre con similares niveles de calidad del que están empleando actualmente o bien porque desconfían que haya empresas detrás de las aplicaciones disponibles que garanticen el mantenimiento y soporte de dichos programas.

Particularmente, varios gobiernos de países desarrollados y en desarrollo han fomentado la adopción de OSS en las organizaciones, a través del uso de diversas estrategias (Mukerji \& Palanisamy, 2012: Qu et al, 2011; Yaseen \& Bahari, 2014; Heili \& Assar, 2009). De esta manera, se ha hecho gran énfasis en la literatura de OSS en estudiar su adopción y experiencias a nivel organizacional.

Adicionalmente, para Galvis (2008) a pesar de que el desarrollo en tecnología informática avanza de manera acelerada, hay tendencia de algunos sistemas ERP (por sus siglas en inglés,
Enterprise Resource Planning) en software libre. Estos sistemas son una manera de automatizar e integrar diferentes procesos de la empresa y acceder a la información en tiempo real, lo cual permite una mejor dirección y reducir la incertidumbre al momento de tomar decisiones. Estos softwares libres cumplen con estándares de calidad internacional y pueden ser analizados e implementados en la pequeñas y medianas empresas de Colombia. Adicionalmente, al igual que los ERP, hay otros softwares como el CRM los cuales hacen que una Pyme sea más competitiva en el mercado y asegure permanencia y vanguardia en sus productos/servicios, pero se hace necesario saber qué hace que las empresas Pyme adopten sistemas OSS, para esto existen unos estudios realizados en varias investigaciones, las cuales deben tener en cuenta las Pyme al momento de implementar software libre que se relacionan en la Tabla 1.

Tabla 1. Relación de factores externos en adopción de OSS entre autores

Table 1. Relationship of external factors in OSS adoption among authors

\begin{tabular}{|c|c|c|}
\hline Investigación & Autor (es) & Factores \\
\hline \multirow{4}{*}{$\begin{array}{l}\text { User acceptance model of open } \\
\text { source software }\end{array}$} & \multirow{4}{*}{$\begin{array}{l}\text { Gallego, Luna, \& } \\
\text { Bueno, } 2008\end{array}$} & 1. La calidad de software \\
\hline & & 2. La capacidad del sistema \\
\hline & & 3. Influencia social \\
\hline & & 4. La flexibilidad del software \\
\hline $\begin{array}{l}\text { Open source software adoption } \\
\text { by South African MSEs: barriers } \\
\text { and enablers }\end{array}$ & $\begin{array}{l}\text { Ellis \& Van Belle, } \\
2009\end{array}$ & $\begin{array}{l}\text { 1. Factores tecnología: costo de software y hardware, } \\
\text { confiabilidad, expectativa de desempeño, condiciones de } \\
\text { facilidad, complejidad. } \\
\text { 2. Factores organizacionales: carácter innovador de las TIC, } \\
\text { experiencia del personal y recursos disponibles. } \\
\text { 3. Factores ambientales: disponibilidad de soporte, legitimidad y } \\
\text { apoyo externo. }\end{array}$ \\
\hline \multirow{7}{*}{$\begin{array}{l}\text { Open Source Software } \\
\text { Adoption: Motivations of } \\
\text { Adopters and Amotivations of } \\
\text { Non-adopters }\end{array}$} & \multirow{7}{*}{$\begin{array}{l}\text { Macredie y } \\
\text { Mijinyawa, } 2011\end{array}$} & 1. Ahorro de costos de licencia \\
\hline & & 2. La funcionalidad \\
\hline & & 3. Comunidad de soporte \\
\hline & & 4. Medios de comunicación Web \\
\hline & & 5. La innovación \\
\hline & & 6. Inversión de capital \\
\hline & & 7. Infraestructura de redes e internet \\
\hline
\end{tabular}

Fuente: elaboración propia. 
En cuanto a la adopción de OSS por parte de las organizaciones, en la literatura existen investigaciones enfocadas principalmente en analizar o identificar factores que promueven la adopción de OSS. Se ha encontrado que organizaciones interesadas en la reducción de las tasas de licencia de software propietario, y con el fin de evitar al mismo tiempo sanciones y responsabilidades legales asociados con su uso ilegal, ven como una alternativa plausible la adopción del Software de Código Abierto (Nagy, Yassin \& Bhattacherjee, 2010). Diversas empresas han reportado un ahorro sustancial en sus costos de operación debido a la adopción de OSS (Sukhoo et al, 2013).

De hecho, numerosos estudios han demostrado que profesionales de oss y organizaciones tienden a apreciar que el OSS es por lo general de forma gratuita (Kamseu \& Habra, 2009; Ven et al, 2008), no obstante esta percepción podría ser errónea dado que no todo el software libre es gratuito (Ven et al, 2008); sin embargo, si se puede afirmar que la filosofía de la tecnología de código libre se presta para hacer que la tecnología disponible para las masas sea adquirida a un costo relativamente bajo en comparación con el software propietario (Karume, \& Mbugua, 2012).

Otros factores que inciden en la adopción de OSS a nivel empresarial son los beneficios técnicos que OSS genera en comparación con el software propietario, tales como la posibilidad de acceder y modificar el código fuente (Morgan \& Finnegan, 2007), ya que permite realizar diversas modificaciones de varias personas y expertos, lo que resulta en una mayor calidad de software (Ven et al, 2008) y en una adecuación a las necesidades de la empresa.

En particular para empresas de mercados emergentes se ha discutido sobre la ventaja competitiva que se puede originar como consecuencia de la adopción de software de código (Urban \& Greyling, 2015), ya que les permite reducir la brecha digital existente con organizaciones de países mucho más desarrollados (Mindel et al, 2007). La cantidad de organizaciones adoptantes de OSS aumenta en el tiempo, ya sea como resultado de una decisión estratégica o simplemente como consecuencia de las opciones tecnológicas (López, Costal, Ralyté, Franch, Méndez \& Annosi, 2016, p. 524; Urban \& Greyling, 2015).

\section{Modelos de aceptación del software de código abierto}

En la literatura se han abordado diversos modelos sobre la adopción de software de código abierto. En particular, la investigación realizada por Li, Tan, Xu \& Teo (2011) expone que la adopción de un sistema de información puede reflejarse a través de dos formas: bien sea por las intenciones de adopción de los no adoptantes o por el grado de adopción de los adoptantes. En este orden de ideas, estos autores, sobre la base de los fundamentos teóricos de la Teoría de la Auto Determinación, proponen y evalúan empíricamente dos modelos conceptuales para examinar las intenciones y el grado de adopción de OSS.

El primer modelo hace referencia a adoptantes del OSS, este incluye la motivación intrínseca y extrínseca. Por su parte, en la motivación intrínseca se distinguen los siguientes tres factores que impulsan la adopción de OSS (Li et al, 2011): saber, lograrlo y experimentar estimulación. La otra parte de este modelo de investigación es la motivación extrínseca que se divide a su vez en tres grupos (Li et al, 2011): regulación externa, regulación introyectada y regulación identificada.

El segundo modelo es el de no adoptantes, Li et al (2011) dividen este modelo en tres aspectos relacionados con la desmotivación: creencias 
de capacidad, creencias de esfuerzo y creencias de estrategia.

Adicionalmente, se ha utilizado ampliamente el Modelo de Aceptación Tecnológica, este potente modelo permite un contraste de comportamiento por parte del usuario y se basa en cuatro variables o constructos fundamentales que son: la Utilidad Percibida (PU), la Percepción de Facilidad de Uso (PEA), la Intención de uso (UI) y el Comportamiento de Uso (UB) (Gallego et al., 2008). En primer lugar, la utilidad percibida se define como el grado en que una persona cree que el uso de un sistema en particular podría mejorar su rendimiento en el trabajo (Davis, 1989, citado en Gallego et al., 2008). En segundo lugar, la percepción de la facilidad de uso se refiere al grado en que una persona cree que el uso de un sistema en particular sería libre de esfuerzo (Davis, 1989, citado en Gallego et al., 2008). Según Davis, la facilidad de uso percibida tiene una influencia directa sobre la utilidad percibida. Estas dos variables son determinadas por elementos externos y tienen una influencia directa en la UI hacia una tecnología específica (Pijpers, 2001 citado en Gallego et al., 2008). A continuación, la intención de utilizar representa la evaluación de los usuarios de la conveniencia de la aplicación y el uso de una tecnología específica (Lederer, Maupin, Sena, y Zhuang, 2000 citado en Gallego et al., 2008). Por último, el comportamiento de uso es una medida de la probabilidad que una persona vaya a utilizar una aplicación específica (Ajzen y Fishbein, 1980 citado en Gallego et al., 2008).

Debido al desconocimiento, existen muchas organizaciones y personas reacias o escépticas a adoptar OSS, por lo que se considera relevante la realización de este estudio con el fin de brindar marco que le sirva de ayuda o apoyo a las futuras Pyme adoptantes de OSS en el contexto social, cultural y económico de las economías emergentes, mostrándoles su uso, beneficios y criterios de selección para la adopción de este tipo de software.

\section{METODOLOGÍA}

El trabajo de investigación está basado el Modelo de Aceptación Tecnológica (TAM), desarrollado por Davis (1989) para predecir la aceptación de los sistemas de información por los usuarios en las organizaciones, adaptándolo con unos factores externos que influyan en la adopción del software libre por medio de gerentes y encargados de sistemas de las Pyme en Medellín. Dicho modelo se ha utilizado para medir adopción de tecnología en estudiantes (Valencia, Benjumea y Rodríguez-Lora, 2014), nuevos empresarios (Montoya, Valencia \& Montoya, 2016), aplicativos móviles (Valencia, González y Castañeda, 2016; Echavarría, Valencia, \& Bermúdez, 2017).

La investigación es de tipo exploratoriodescriptivo con el fin de identificar los factores que llevan a la adopción o no del software libre en las Pyme de la ciudad de Medellín y describir este fenómeno. Es una investigación no probabilística bajo el criterio de ser empresas de este tamaño que implementen en sus procesos algún tipo de software y que lleven en el mercado más de un año para garantizar la trazabilidad del estudio. Para llevar a cabo la investigación, se realiza una revisión literaria en bases de datos de estudios previos en temas relacionados con características de software libre, barreras y ventajas en la implementación de software libre, adopción y uso de software libre en Pyme e impacto de estas en la economía nacional, con el fin de identificar los elementos relacionados en este proceso a nivel mundial y poder tener una guía de los principales factores y variables que influyen en la adopción de software libre a nivel empresarial, sirviendo así como base para la construcción y validación de la entrevista semiestructurada. Esta consulta de literatura 
se hace en las bases de datos con las que cuenta el ITM, entre las que se encuentran Embase, Engineering Village, Reaxys, Science Direct y Scopus y en revistas de gran impacto a nivel internacional y con alta calidad académica como European Journal of Information Systems, Telecommunications Policy y Data Base for Advances in Information Systems.

Como medio de recolección de los datos se hacen entrevistas semiestructuradas, cara a cara, a cinco gerentes y jefes de sistemas de diferentes empresas Pyme de la ciudad de Medellín (ver Tabla 2) a los cuales se les da el consentimiento informado que no se publicara sus nombres ni el de las empresas que representan. Las preguntas realizadas a estas personas son formuladas a partir de los constructos del Modelo de Aceptación Tecnológica (TAM), con preguntas abiertas, y se han ampliado a partir de los hallazgos de otras variables y factores preponderantes al momento de tomar la decisión de adoptar software libre en el contexto organizacional. Las entrevistas que se realizaron fueron grabadas y transcritas para proceder a su respectivo análisis (Fernández, 2006). EI análisis de los datos recolectados, después de transcritos, se revisaron y se realizó una codificación en el cual se agrupa la información obtenida en categorías que concentran las ideas, conceptos 0 temas similares descubiertos por el investigador, o los pasos o fases dentro de un proceso y así tener más facilidad para ver su relación (Rubin y Rubin, 1995 citado en Fernández, 2006), de acuerdo al modelo TAM, contrastando entre cada entrevistado qué relación guardan y luego la relación existente entre cada código o constructo del modelo seleccionado.

Después de la consulta literaria que se hizo sobre el tema a investigar, se encuentran variables externas como son el apoyo gubernamental, planteada en la investigación de Ortega (2016) y la puesta en marcha en planes del Gobierno nacional, calidad del software, cultura tecnológica de la organización, infraestructura tecnológica, disponibilidad de personal con cocimiento técnico, que según su relación se conectaron con los constructos del Modelo de Aceptación Tecnológica TAM y fueron agregadas en el modelo modificado (ver Fig. 1). Estas relaciones y la importancia de cada variable fueron validados y retroalimentados a partir de la opinión de los expertos interrogados en el proceso de recolección de información.

Tabla 2. Entrevistas y sector de la empresa entrevistada

Table 2. Interviews and sector of the company interviewed

\begin{tabular}{ll}
\hline \multicolumn{1}{c}{ Entrevista } & \multicolumn{1}{c}{ Sector de la empresa entrevistada } \\
\hline Entrevista 1 & $\begin{array}{l}\text { Sector de transporte masivo de pasajeros en modalidad de } \\
\text { alimentación }\end{array}$ \\
\hline Entrevista 2 & Sector de colchonería y ropa hogar \\
\hline Entrevista 3 & Sector de tecnología en diseño de software y soluciones en TIC \\
\hline Entrevista 4 & $\begin{array}{l}\text { Sector de tecnología en diseño de software a la medida al sector } \\
\text { salud }\end{array}$ \\
\hline Entrevista 5 & $\begin{array}{l}\text { Sector de tecnología en elaboración de productos para el control } \\
\text { de flota }\end{array}$ \\
\hline
\end{tabular}

Fuente: elaboración propia. 


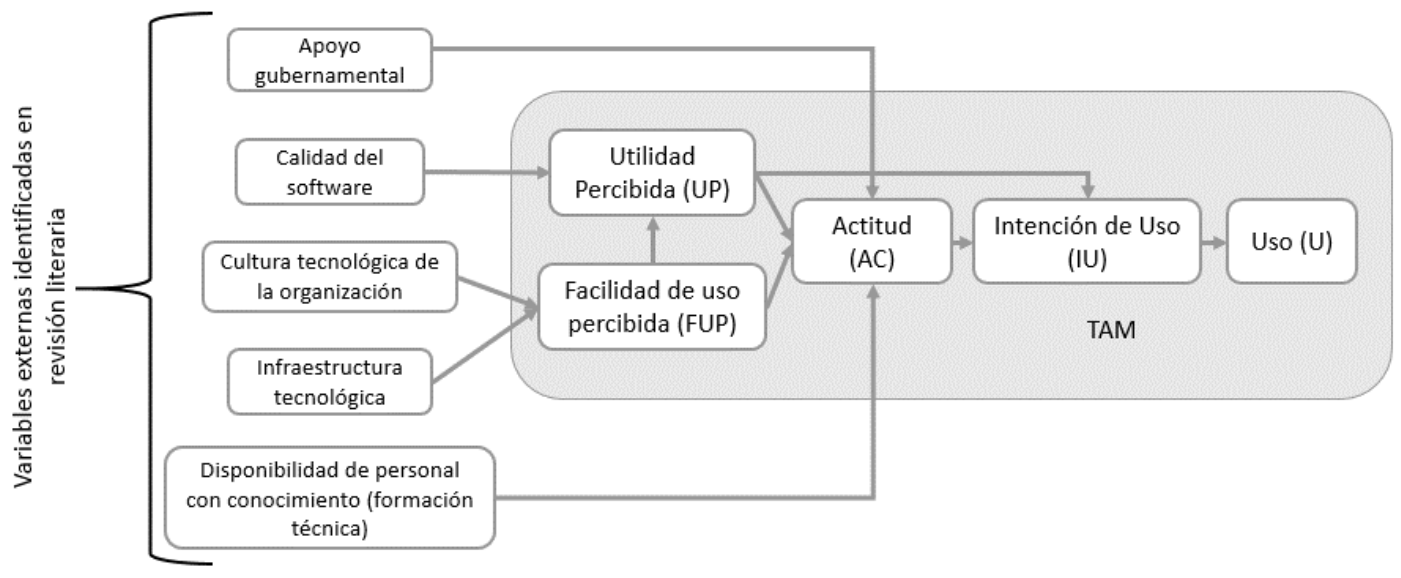

Figura 1. Modelo de variables para adopción de software libre en Pymes

Figure 1. Model of variables for free software adoption in SMEs. Own elaboration based on the TAM model Fuente: elaboración propia apoyado en el modelo TAM.

\section{RESULTADOS Y DISCUSIÓN}

Después de la realización de las entrevistas; se procede con el análisis con base al Modelo de Aceptación Tecnológica.

En la Fig. 2, se observa que la adopción del software libre en las Pyme de Medellín está en gran parte condicionado por el conocimiento o la capacitación que tenga el personal, ya que como indica uno de los entrevistados.

"Los empleados están dados al sistema operativo licenciado, como es Windows. Entonces digamos que los que perciben o los que adoptan el software libre son muy técnicos, entonces a los técnicos les da facilidad, pero a una persona administrativa si le pones un sistema operativo que sea software libre no lo va a adoptar, genera cierta complejidad. Entonces para una persona que tenga una formación técnica creo que es más fácil» (Entrevista 3).

Las empresas entrevistadas dividen entonces a su personal entre administrativo (que no tienen conocimiento técnico del software libre) y personal de sistemas o soporte (que ya tienen conocimientos previos sobre el uso de software libre), aduciendo que el proceso se hace más complejo cuando el personal está acostumbrado a la utilización de herramientas más gráficas y licenciadas que a una que no tenga estas mismas características así le sea de más utilidad o le genere mayor beneficio. 


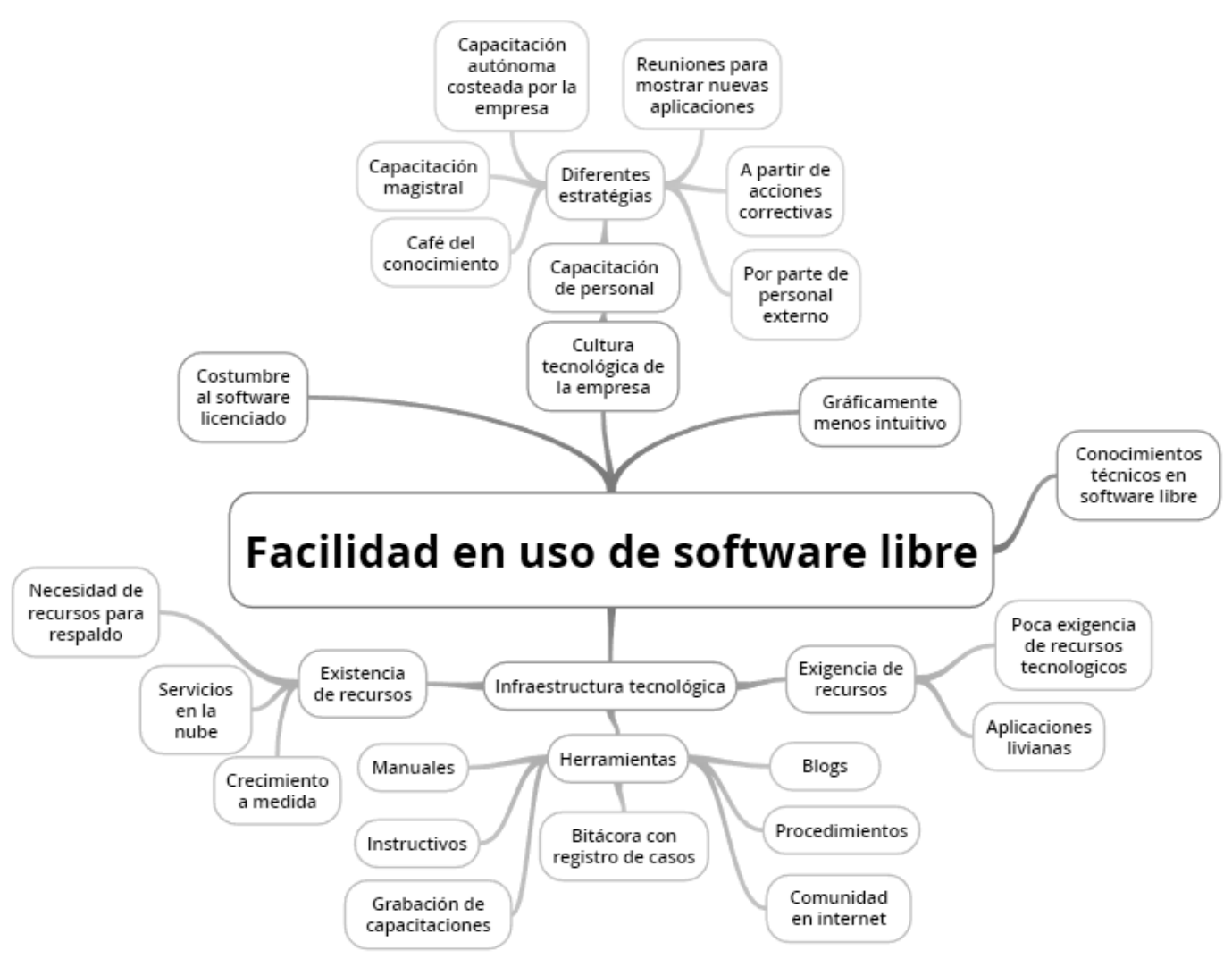

Figura 2. Facilidad de uso de software libre

Figure 2. Ease of use of free software

Fuente: elaboración propia.

Esto lleva a que se haga énfasis en el tema de capacitación de personal y ha llevado a que las empresas tengan diferentes formas de fomentar la cultura tecnológica utilizando estrategias, como: café del conocimiento, capacitaciones magistrales, patrocinar certificaciones que los empleados deseen adquirir, contratación de terceros para capacitaciones, entre otras.

Plantean también que esta facilidad de uso se da de igual forma cuando la empresa cuenta con las herramientas e infraestructura adecuadas para su funcionamiento, esto ha llevado a que se le dé importancia a la documentación del software libre, donde han surgido diferentes técnicas para poder conservar ese conocimiento y garantizar la perdurabilidad y estabilidad de los sistemas. Se encuentra entonces que una de las empresas, al momento de la implementación del software libre, determinó que se debían grabar todas las capacitaciones dictadas sobre el funcionamiento y soporte, y estas son consultadas cada que ingresa personal nuevo que vaya a ser uso de ellas. Otras, por ejemplo, guardan bitácoras de los casos ocurridos en los diferentes sistemas. "También hay secciones de fallas que han sucedido en la plataforma y se tiene documentación de eso para cuando a alguien le suceda lo pueda consultar y encontrar fácilmente la solución» (Entrevista 4). Este tipo de estrategias han ayudado a que los problemas ocurridos con el software libre sean solucionados con mayor rapidez, ya que cuentan con las herramientas necesarias para dar el debido soporte. 
Referente al tema de infraestructura se ha resaltado una de las características del software libre y es que no exige a la empresa mayor inversión en infraestructura tecnológica, lo cual facilita que las empresas adopten este tipo de herramientas y se conviertan en un aliado al momento de adquirirlas. Esto se traduce en que las empresas entrevistadas cuenten con la infraestructura adecuada para soportar los requerimientos de sus diferentes aplicativos libres, pero de esta misma manera se es consciente que la empresa debe considerar dentro de su infraestructura el respaldo que permita dar respuesta ante algún imprevisto "en ese tema de capacidades hay que tener mucho cuidado en no tener lo mínimo y al ras como que se quede la plataforma, pero tampoco sobreestimar los recursos de la empresa, de tener recursos sin utilizar» (Entrevista 4).

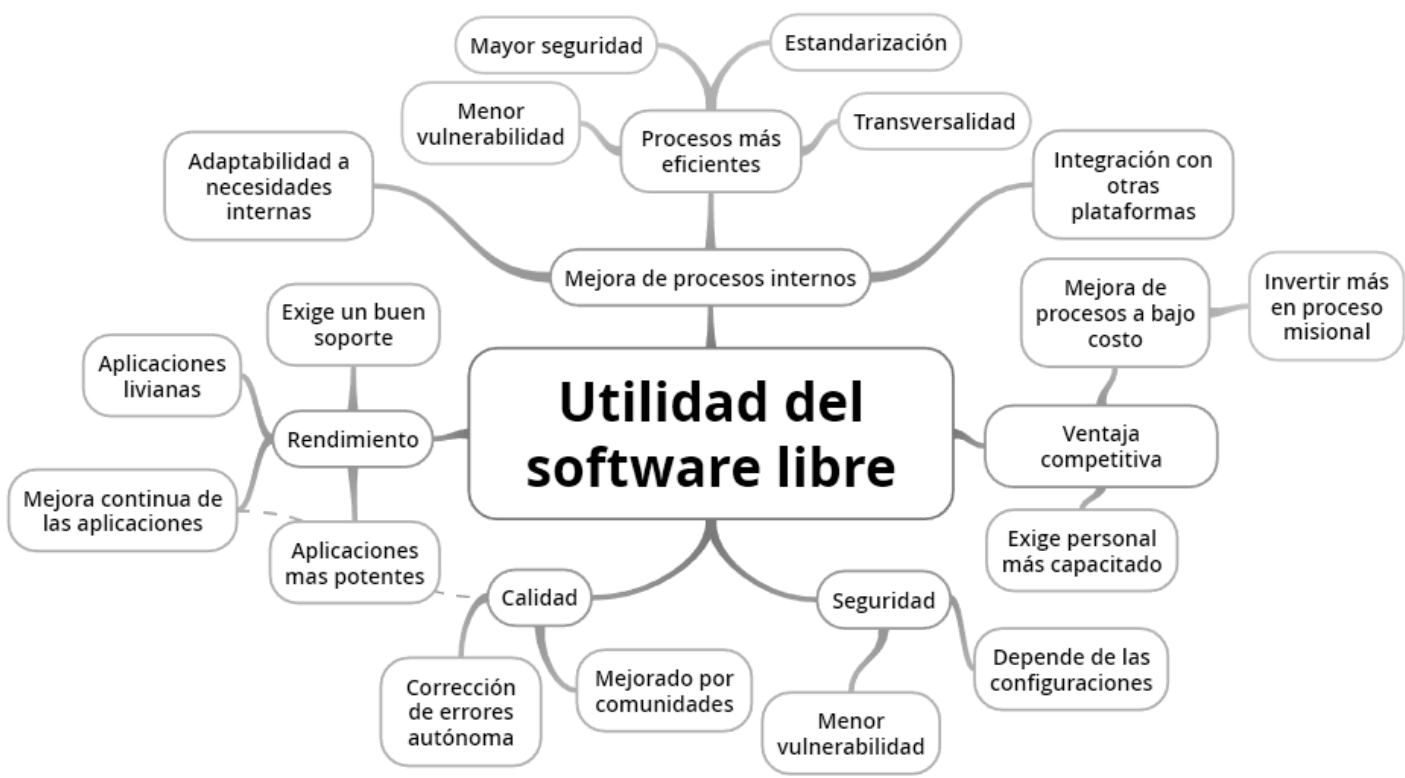

Figura 3. Utilidad de software libre

Figure 3. Free software utility

Fuente: elaboración propia.

En la Fig. 3, se muestra la importancia que las empresas entrevistadas le han dado a sus diferentes sistemas y han depositado confianza en el uso de las herramientas libres al utilizarlas de manera transversal en toda la organización y en procesos críticos, incluso han diseñado sus procesos con base a estos sistemas; por ejemplo, una de las empresas entrevistadas que tomó la decisión de utilizar un software ERP libre dice "a través de los procesos que se diseñan al usar el software se han ido estandarizando más los procesos dentro de la organización» (Entrevista 2). Este tipo de sistemas permite que los procesos internos se estandaricen y se tenga mayor seguridad en la información, teniendo en cuenta que cada empresa puede aplicar las configuraciones de seguridad necesarias para su sistema al igual que se disminuye el riesgo y la vulnerabilidad en posibles ataques. Cuando la empresa decide adoptar diferentes sistemas libres se da la opción de poder integrar estas plataformas, es 
el caso de una empresa entrevistada que adoptó SugarCRM y lo integró con su cuenta de correo libre llamada Thunderbird: "el correo electrónico que es libre, se integra muy bien con el SugarCRM, entonces manda las alertas cuando se asigna un caso a una persona, facilita mucho mirar el histórico, ver el progreso de las ordenes de producción, indicadores» (Entrevista 5).

De otro lado, la utilización de software libre exige personal capacitado, lo cual se traduce a que se tenga una mejor postura y respuesta a las exigencias del mercado en cuanto a plataformas e infraestructura tecnológica se refiere; en el medio actual no es importante tener la herramienta tecnológica, sino saber gestionarla para alcanzar la ventaja competitiva, "el software libre hace que el personal se capacite y explore otras opciones y alternativas, entonces genera que la empresa esté más preparada para ajustarse a la necesidad de un cliente cuando le ha tocado trabajar en diferentes entornos» (Entrevista 5).

Otra utilidad para las empresas identificada en las entrevistas es que ofrece un alto rendimiento en las actividades de la empresa Si cumple con todos los requerimientos de una empresa, y de hecho yo creo que no hay empresa en estos momentos que algún servicio lo utilice bajo Linux, sobre todo por la confiabilidad, seguridad, lo robusto, a pesar de que es un sistema liviano de instalar, es algo muy muy robusto y muy completo (Entrevista 1).

Es así como las empresas entrevistadas perciben las aplicaciones libres como más potentes y más livianas, teniendo en cuenta que todo sistema puede tener sus fallas $y$ requiere de soporte; es necesario, entonces, contar con personal capacitado que esté en las condiciones de dar respuesta a requerimientos internos que surjan en la operación. Otra percepción que se encuentra de las utilidades del software libre es la calidad y la seguridad del sistema, en el tema de seguridad las empresas entrevistadas coinciden al decir que tener software libre, especialmente los sistemas operativos, se tiene menos riesgo o vulnerabilidad de ataques externos, pero que esto depende en gran medida de la configuración y medidas de prevención que se den. Por otra parte, la calidad. Las personas tienen la opción de hacer correcciones inmediatas, adicional a que se pueden encontrar mejoras en las versiones, gracias al aporte que se hace por medio de las comunidades a nivel internacional que aportan al crecimiento de determinado programa.

Sobre el contexto de la ciudad de Medellín, en la Fig. 4 se ha encontrado mayor adopción y uso de software libre dentro de las empresas en los procesos de soporte y desarrollo, ya que estas son las áreas donde está el personal con mayor nivel de formación en TIC de la empresa. Caso contrario pasa en las áreas administrativas y más cuando son sistemas de uso cotidiano como herramientas ofimáticas que son más sensibles según los entrevistados, a las personas que están acostumbrados a determinado paquete ofimático, "en algunos casos es más fácil un software licenciado, puntualmente por el office, si una persona que usa office se cambia a open office es difícil porque es una estructura diferente, no es imposible, pero sí difícil y sensible» (Entrevista 5). 


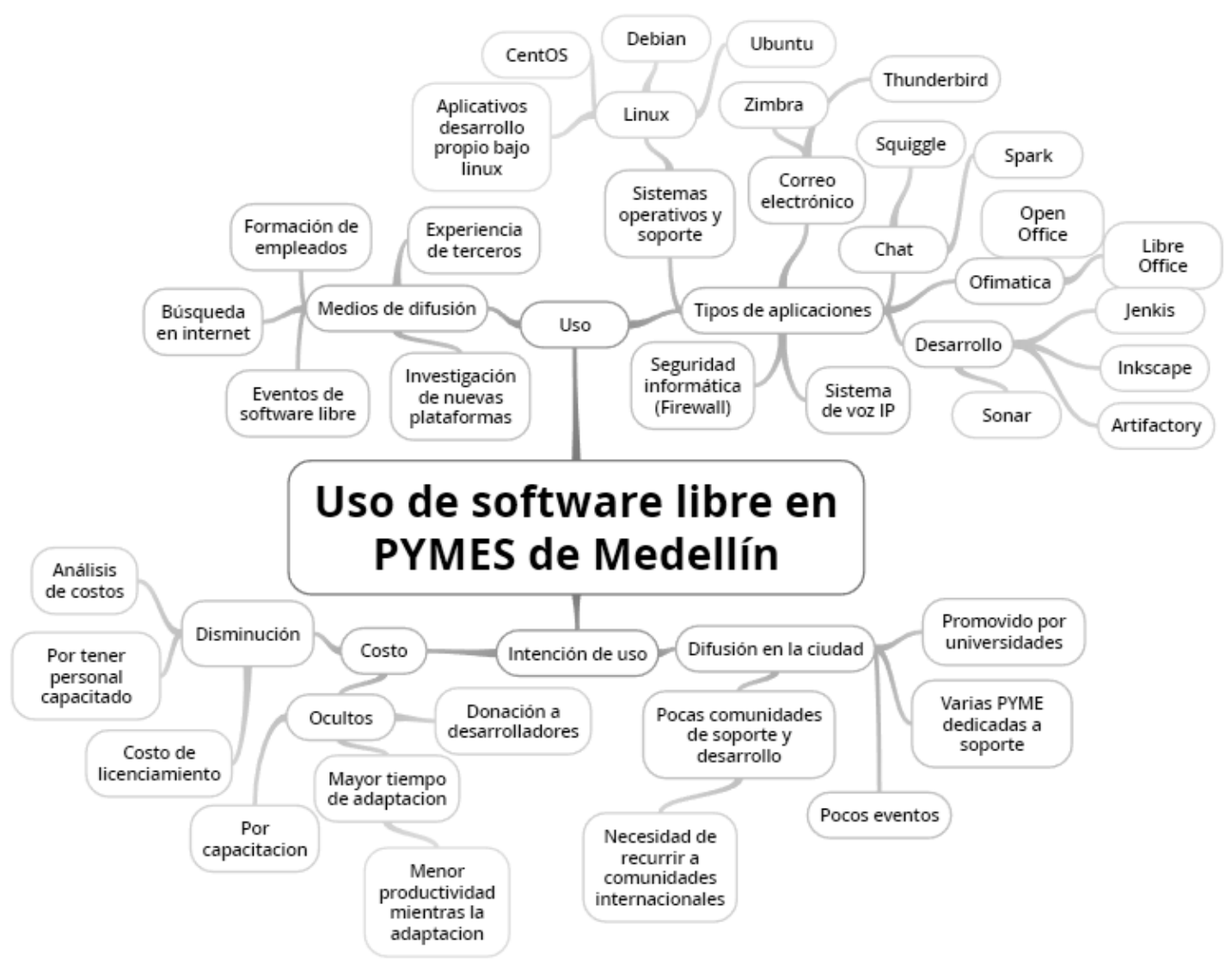

Figura 4. Uso de software libre en Pyme de Medellín

Figure 4. Use of free software in SMEs in Medellín

Fuente: elaboración propia.

Muchas de las empresas entrevistadas han adoptado el software libre por la misma formación del empleado, en donde por su experiencia laboral y formación académica han conocido los diferentes sistemas libres, sus beneficios y soporte y han logrado tomar esta decisión de implementarlo en procesos de la compañía. En las entrevistas realizadas se encuentra que se han utilizado diferentes medios para encontrar el software libre ideal: «búsquedas en internet, consultas en colaboración con terceros en otros lugares del mundo. Yo fui partner de ERP dos años en Colombia y a partir de ahí fue que se fortaleció la idea implementar open ERP en la compañía» (Entrevista 2). Se encuentra en esta empresa, como en varias entrevistadas, que se han apoyado en las búsquedas en internet y en las experiencias de terceros con el software que le han servido para tomar la decisión de adopción.

Así pues, las empresas han visto el tema de la reducción de costos como un beneficio y como un impulso que los lleva a la intención de usar este tipo de sistemas, aunque es importante tener el personal capacitado que pueda dar soporte al sistema, ya que en las empresas entrevistadas ninguna tomó la decisión de tercerizar el proceso de tecnologías de la información y comunicación. Este personal capacitado, y el tiempo de adaptación a la empresa han determinado un costo oculto que se evidencia en el tiempo requerido para adaptar el software a la organización y se deje entonces de emplear el tiempo en otras 
funciones propias del negocio: "el tiempo necesario para su implementación se representa en costo económico inherente, que no es muy visible, pero si una persona se queda $x$ tiempo solucionando algo, está dejando de emplearlo en otras actividades al cliente» (Entrevista 4).

Según los hallazgos resumidos en la Fig. 5, las empresas entrevistadas han encontrado varias ventajas al utilizar software libre en vez de privativo en las empresas; el más común es el ahorro de costos, "Costos. Solamente para administrar y dar seguridad es un tema de costos, porque permite tener ciertas herramientas y flexibilidad de configuración que otras licenciadas requieren un costo adiciona/» (Ent.3). Esto ha significado para las empresas entrevistadas aprovechar los ahorros en costos de licenciamiento para invertir más en el CORE del negocio. Este tipo de sistemas permite que la empresa tenga autonomía en las modificaciones y soporte de sus aplicaciones libres por tener acceso al código fuente; poder adaptarlo a los requerimientos generados sin tener que incurrir en costos adicionales y no depender de un tercero para realizar las modificaciones necesarias: "adicionalmente que no dependemos de la velocidad de un tercero, sino que muchas cosas las podemos resolver in house» (Entrevista 2).

Se ha encontrado, además, que las empresas entrevistadas tienen una percepción de seguridad superior en el software libre, y por este motivo han montado su sistema de seguridad informativa basados en Linux, al igual que perciben una potencia superior al software licenciado.

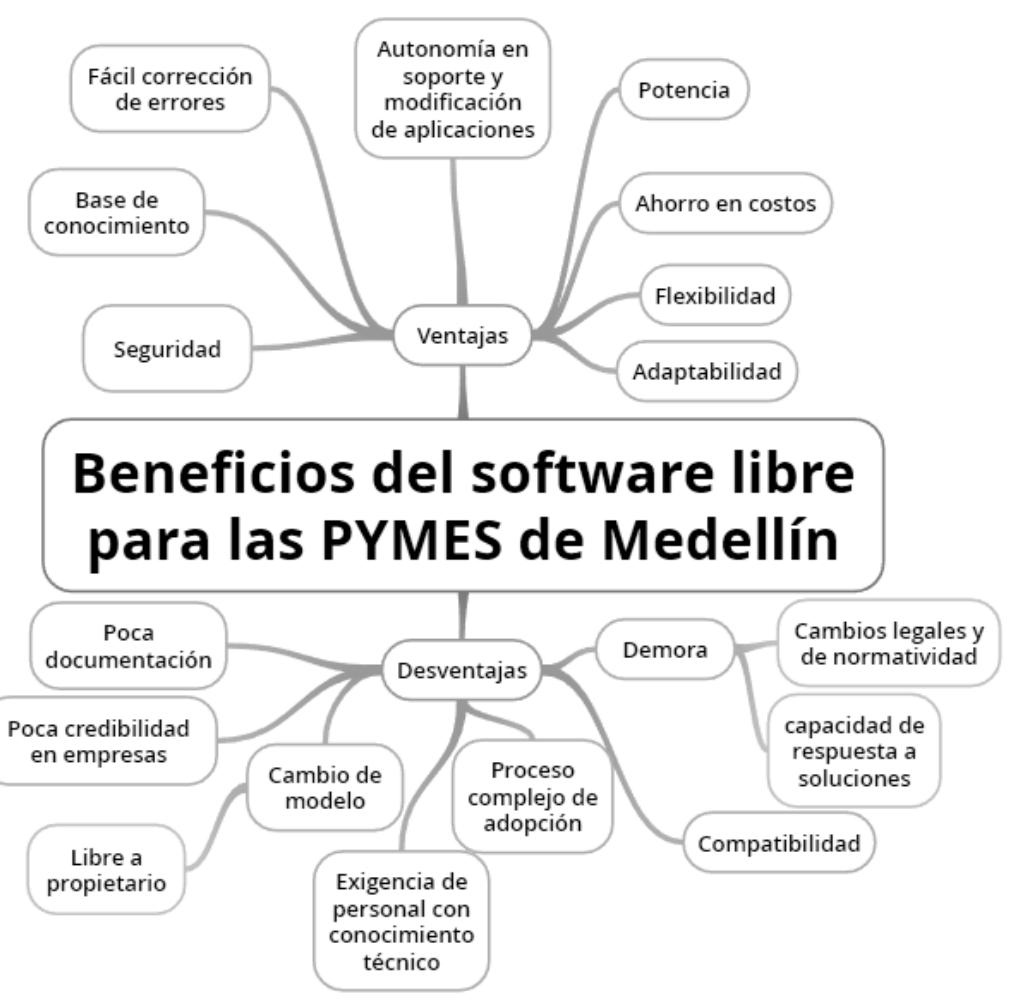

Figura 5. Beneficios del software libre para las Pyme de Medellín

Figure 5. Benefits of free software for SMEs in Medellín

Fuente: elaboración propia. 
Así, las empresas han encontrado también ciertas desventajas en el uso del software libre; una de ellas es que el modelo de licenciamiento de estas plataformas puede ser modificado y tener que incurrir en costos de licenciamientos a futuro.

Es el caso de open ERP que empezó como un software libre y hoy ya un $70 \%$ del software se ha comenzado a ser privativo, entonces hay que pagar. Uno cree en la plataforma, se pasa y en cualquier momento las actualizaciones dejarían de darse porque la casa matriz o creadores ven que no están recibiendo los suficientes ingresos y cambian el modelo del proyecto (Entrevista 2).

Se encuentra que las empresas ven una desventaja en la compañía por la falta de credibilidad y apoyo directivo para el uso de software libre. Esto puede llevar a que los directivos de sistemas tengan que prescindir de este tipo de plataformas. Cuando los sistemas son desarrollados en otros países, se encuentran con una barrera, consistente en el tema de localización de los sistemas y el cambio en normatividad, ya que en algunos casos las empresas no cuentan con el personal disponible para hacer estas modificaciones, sin tener que recurrir a comunidades internacionales o a los creadores del software: "los desarrolladores externos a la empresa no le hicieron las modificaciones, como tipo normatividad en NIFF, impuestos como CREE, entre otros asuntos contables y legales que se debe contar con la base suficiente para poderle hacer actualizaciones al software» (Entrevista 2).

Se hace notoria esta desventaja al momento de dar respuesta a determinados errores, ya que es necesario realizar un proceso de consulta en los diferentes medios y es complejo al tener un software libre dentro de un proceso crítico, precisamente por esa capacidad de respuesta ante fallas:

Cuando son ambientes muy críticos, el tema del soporte también se vuelve muy crítico, cuando se monta una plataforma que tiene que estar 24/7 disponible; muchas veces cuando tienen software libre, por detrás están las comunidades y la ayuda que es muy buena pero se queda corta, porque sí o sí se debe solucionar en un corto periodo de tiempo, porque no hay manera de esperar, entonces en ese caso, cuando se adquiere un software licenciado con soporte el soporte, es más rápido porque se está pagando es por eso (Entrevista 4).

La Fig. 6 resume los resultados frente a las condiciones de uso de software libre en PYME, encontrándose que es importante el tema de disponibilidad de personal y apoyo gubernamental. En el primero se pudo observar que las empresas cuentan con personal capacitado dentro de un área de soporte o desarrollo que cuentan con un nivel alto en formación en TIC; para estas áreas es fácil el proceso de adopción y uso del software libre ya que cuentan con la base de conocimiento necesaria para utilizarlo de forma adecuada y eficiente. Caso contrario se encuentra en el momento en que el software va a ser utilizado por personal administrativo $u$ operativo donde, por lo general, no tienen las bases para su utilización y están acostumbrados a plataformas más gráficas o propietarias y les genera más dificultad adoptar este tipo de tecnologías. Así pues, la adopción de software libre está condicionado en la empresa por personal capacitado o con conocimiento en estas plataformas que puedan dar soporte o modificaciones al momento de requerirlo y puedan brindar capacitación y acompañamiento a usuarios 
finales del software libre: "Desde que a los usuarios se les enseñe, ellos aprenden fácilmente y adoptan los conocimientos que uno les brinda. Hay que estar reforzando mucho las capacitaciones que se hacen para que no se vayan a caer los procesos» (Entrevista 3).

El segundo factor es el apoyo gubernamental que se encontró en las empresas entrevistadas, que no recurren a programas o ayudas que brinda el Gobierno para facilitar el proceso de adopción de estas tecnologías. Hace falta reformar la interacción entre Gobierno, universidad y empresa para fortalecer procesos de competitividad de la ciudad y del país. Se encontró, además, que las empresas entrevistadas donde su CORE de negocios son las tecnologías y el soporte, han acudido con mayor frecuencia a entidades como Ruta $\mathrm{N}$ (en procesos de innovación y apoyo de conocimiento especializado), entidades educativas (por el conocimiento y experiencia en desarrollo de aplicaciones) y Colciencias (en el tema de impulso financiero $y$ acompañamiento de proyectos) para impulsar los proyectos tecnológicos que se encuentran desarrollando en su entorno organizacional.

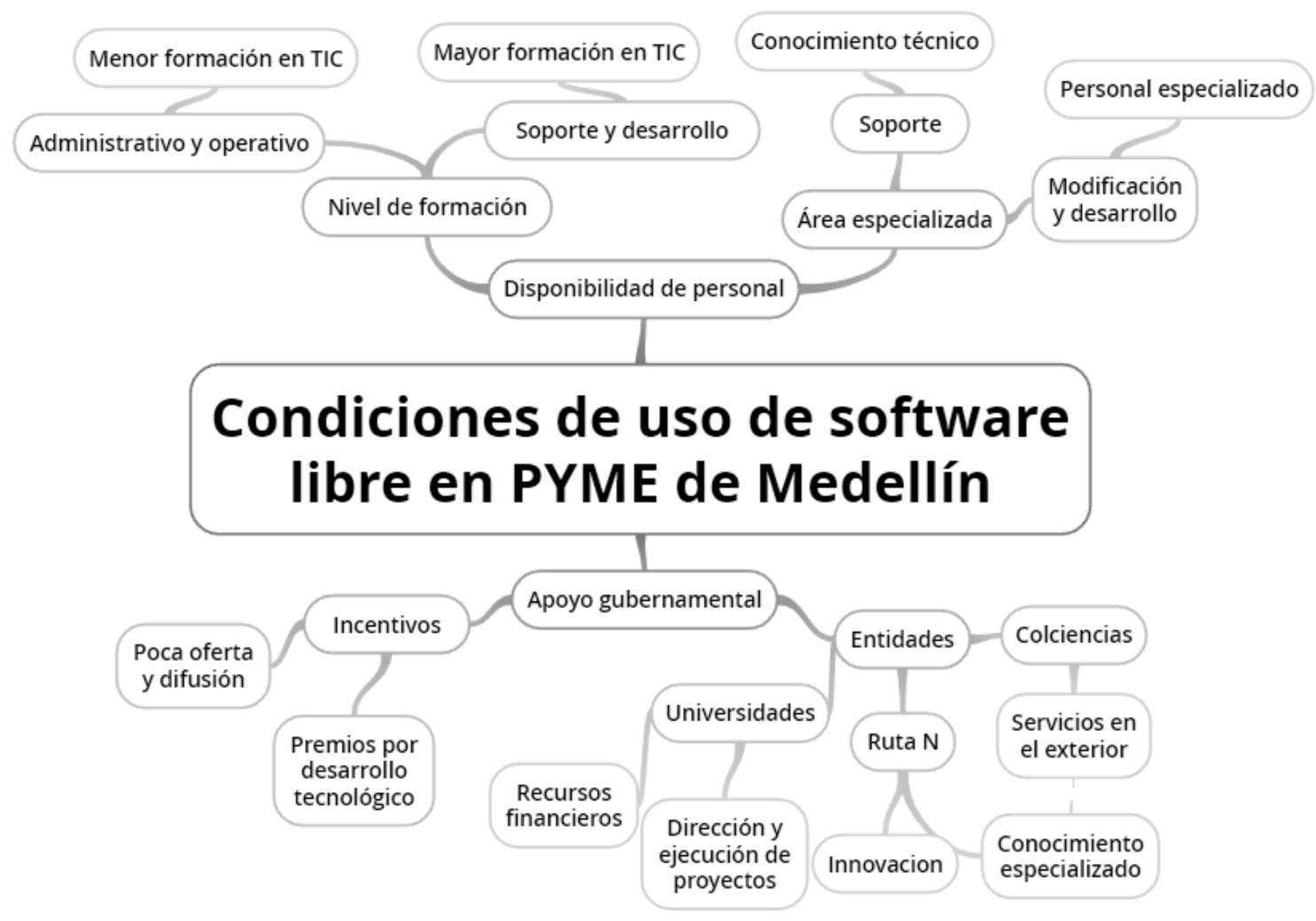

Figura 6. Condiciones de uso de software libre en Pyme de Medellín

Figure 6. Conditions of use of free software in SMEs in Medellín Fuente: elaboración propia. 


\section{CONCLUSIONES}

Como resultado de la investigación, se logran identificar los factores que hacen que las empresas Pyme de Medellín entrevistadas determinan la adopción del software libre, encontrando que el modelo propuesto cumple los resultados de la investigación. Se observa que la cultura tecnológica de la organización y su infraestructura son factores que determinan la facilidad de su uso. Adicionalmente, se observa que la calidad del software es un determinante para percibir la utilidad del software libre, y que tanto el apoyo gubernamental como la disponibilidad de personal capacitado en TIC son factores que influyen en la actitud que toma la empresa frente al uso de estas tecnologías libres.

Se logran identificar, después de la revisión literaria, los factores que determinan la adopción del software libre en las Pyme, encontrando que el apoyo del Gobierno es importante al momento de impulsar tecnologías dentro de una organización, de manera directa o por medio de entidades especializadas en el tema, pero en relación con las entrevistas realizadas, se encuentra que estas empresas no hacen uso de estos programas y entendidas públicas especializadas en Medellín. Es relevante para las Pyme entrevistadas contar con personal con conocimiento técnico, que les permita hacer un uso efectivo de las plataformas, así se garantiza la estabilidad del sistema y por tal la perdurabilidad del mismo, al igual que una infraestructura base para soportar las diferentes aplicaciones, aunque se tiene la percepción que no exige mayores especificaciones. Por último, se ha reconocido las ventajas del software libre, siendo común en los documentos consultados la percepción positiva en costo, calidad y seguridad de los sistemas libres en términos generales.
Las empresas entrevistadas perciben la adopción del software libre como un proceso que requiere un esfuerzo que permita la implementación y uso de estos sistemas, por lo cual, de todos los factores identificados, la capacitación es el elemento que los empleados requieren con mayor prioridad para la adopción de software libre; la costumbre a la interfaz de sistemas licenciados ha creado un esquema que hace que el personal sea reacio al cambio, sin embargo, se identifica que cuando la empresa fortalece la capacitación se logra adoptarlo de manera eficiente. De igual forma, se ha identificado que las áreas de las empresas entrevistadas, donde más se usa el software libre son las áreas de soporte y desarrollo, donde se identifica una fácil asimilación de estas tecnologías por su conocimiento técnico. A nivel directivo, se encuentra que estas tecnologías son utilizadas gracias a los beneficios que encuentran en ella como ahorro de costos, base de conocimiento, seguridad, calidad y adaptabilidad a necesidades internas, elementos que, según los entrevistados, es difícil encontrar en un sistema propietario.

Actualmente, se ve la necesidad en las empresas Pyme de mejorar su competitividad para garantizar su permanencia en el mercado, de allí el beneficio de utilizar herramientas de bajo costo que ayuden con este objetivo. Por esto, es importante garantizar en el proceso de adopción un personal con el conocimiento básico en estas plataformas que facilite el soporte y la adaptabilidad del mismo a los requerimientos de la empresa. Estructurar un fuerte plan de capacitaciones que permita a los empleados sumergirse en el uso de software libre y adopten estas herramientas $y$, por último, buscar con gestores tecnológicos $y$ entidades educativas la ayuda para impulsar la implementación de estas herramientas en los procesos internos que permitan generar ventaja competitiva del negocio. 


\section{REFERENCIAS}

Alexy, O., Henkel, J., \& Wallin, M. W. (2013). From closed to open: Job role changes, individual predispositions, and the adoption of commercial open source software developmEntrevista Research Policy, 42(8), 1325-1340.

Bandi, S., Kanamadi, S., \& Angadi, M. (2016). Application of Free and Open Source Software (FOSS) towards Responsive Library Services: A Case Study. En International Symposium on Knowledge and Communication. Maharashtra. India.

Bonet, S., Instituto, B. A.-, \& Metalmecánico, T. (2007). Problemas detectados en la difusión del Software Libre en las empresas.

D’MacRedie, R. D., \& Mijinyawa, K. (2011). A theory-grounded framework of Open Source Software adoption in SMEs. European Journal of Information Systems, 20(2), 237-250. http://doi.org/10.1057/ejis.2010.60

Chavarria, L. D., Valencia, A., \& Bermúdez, J. (2017). Agent-based Model for the Analysis of Technological Acceptance of Mobile Learning. IEEE Latin America Transactions, 15(6), 1121-1127.

Ellis, J., \& Van Belle, J.-P. (2009). Open Source Software Adoption by South African MSEs: Barriers and Enablers. Proceedings of the 2009 Annual Conference of the Southern African Computer Lecturers Association, 4149.

http://doi.org/http://doi.acm.org/10.1145/ 1562741.1562746

Fernández Núñez, L. (2006). Fichas para investigadores ¿Cómo analizar datos cualitativos? Bulletí LaRecerca, 1-13. http://doi.org/ISSN: 1886-1946 / Depósito legal: B.20973-2006

Gallego, M. D., Luna, P., \& Bueno, S. (2008). User acceptance model of open source software. Computers in Human Behavior, 24(5), 2199-2216. http://doi.org/10.1016/j.chb.2007.10.006

Gálvez E. J., Riascos, S. C., \& Contreras, F. (2014). Influencia de las tecnologías de la información y comunicación en el rendimiento de las micro, pequeñas y medianas empresas colombianas. Estudios Gerenciales, 30(133), 355-364.

Galvis, R. (2008). El aporte económico de las Pymes en Colombia y su actualización tecnológica a partir del software libre basado en el concepto ERP. Entramado, $4(1), 64-79$.

Garmabaki, A., Ahmadi, A., Mahdavi, I., \& Ahmadi, M. (2015). Reliability modeling of open source software based on adoption behavior under stochastic environmEntrevista In: European Safety and Reliability Conference: 06/09/2015 (pp. 3995-3999). CRC Press.

Hoyos, J. \& Valencia, A. (2012). El papel de las TIC en el entorno organizacional de las pymes. TRILOGÍA Ciencia Tecnología Sociedad, (7), 105-122.

Kagiri, T. M., He, Y., \& Henglin, S. (2013). Factors limiting OSS adoption. Oulun, Finland: University of OULU.

Kamseu, F., \& Habra, N. (2009). Adoption of open source software: Is it the matter of quality. PReCISE. Computer Science Faculty, University of Namur, Belgium. 
Karume, S. M., \& Mbugua, S. (2012). Trends in adoption of open source software in Africa. Journal of Emerging Trends in Computing and Information Sciences, 3(11), 1509-1515.

Lakka, S., Michalakelis, C., Varoutas, D., \& Martakos, D. (2012). Exploring the determinants of the OSS market potential: The case of the Apache web server. Telecommunications Policy, 36(1), 51-68.

Li, Y., Tan, C. H., Xu, H., \& Teo, H. H. (2011). Open source software adoption: motivations of adopters and amotivations of non-adopters. ACM SIGMIS Database: the DATABASE for Advances in Information Systems, 42(2), 76-94.

López, L., Costal, D., Ralyté, J., Franch, X., Méndez, L., \& Annosi, M. C. (2016, June). OSSAP-A Situational Method for Defining Open Source Software Adoption Processes. In International Conference on Advanced Information Systems Engineering (pp. 524539). Springer, Cham.

Méndez, L. (2016). Towards a Reference Framework for Open Source Software Adoption. Proceedings of CAISE 2016 Doctoral Consortium. Ljubljana, Slovenia.

Montoya, I., Valencia, A., \& Montoya, A. (2016). Mapeo del campo de conocimiento en intenciones emprendedoras mediante el análisis de redes sociales de conocimiento. Ingeniare. Revista Chilena de Ingeniería, 24(2), 337-350.

Morgan, L., \& Finnegan, P. (2007). How perceptions of open source software influecne adoption: an exploratory study. Proc. 15th European Conf. Information Systems (ECIS 07), Univ. of St. Gallen, 2007, pp. 973-984.
Roca, M. (2007). Software Libre: empresa y administración en España y Cataluña (Vol. 1). Editorial UOC.

Ruiz, C. A. O. (2014). Inclusión de las TIC en la empresa colombiana. Suma de Negocios, 5(10), 29-33.

Shaikh, M. (2016). Negotiating open source software adoption in the UK public sector. Government Information Quarterly, 33(1), 115-132. doi: 10.1016/j.giq.2015.11.001

Silic, M., \& Back, A. (2016). The influence of risk factors in decision-making process for open source software adoption. International Journal of Information Technology \& Decision Making, 15(01), 151-185.

Urban, B., \& Greyling, B. C. (2015). Open source software adoption and links to innovation performance. International Journal of Technological Learning, Innovation and Development, 7(3), 261-278.

Uribe, J. L. (2007). Estudio sobre la situación actual del uso de software libre en algunas empresas de Medellín. PhD Proposal, 1, 86. http://doi.org/10.1017/CBO978110741532 4.004 .

Valencia, A., Benjumea, M \& Rodríguez-Lora, V. (2014). Intención de uso del e-learning en el programa de administración tecnológica desde la perspectiva del modelo de aceptación tecnológica. Revista Electrónica Educare, 18(2), 247-264.

Valencia, A., González, G. \& Castañeda, M. (2016). Structural equation model for studying the mobile-learning acceptance. IEEE Latin America Transactions, 14(4), 1988-1992. 
Varela, L. A. Y., Tovar, L. A. R., \& Chaparro, J. (2010). Modelo de Aceptación Tecnológica (TAM): un estudio de la influencia de la cultura nacional y del perfil del usuario en el uso de las TIC. Innovar, 20(36), 187-203

Ven, K., Verelst, J., \& Mannaert, H. (2008). Should you adopt open source software? IEEE software, 25(3), 54-59.
Whitmore, A., Choi, N., \& Arzrumtsyan, A. (2009). Open source software: The role of marketing in the diffusion of innovation. Information Technology and Control, 38(2).

Yohai, A. (13 de junio de 2013). Las Pyme son las más rezagadas en el uso de las tecnologías de la información: CCIT. (C. Radio, Entrevistador). 